\title{
Generalised reduction of putative endothelial progenitors and CXCR4-positive peripheral blood cells in type 2 diabetes
}

\author{
C. G. Egan • R. Lavery • F. Caporali • C. Fondelli • \\ F. Laghi-Pasini $\cdot$ F. Dotta $\cdot$ V. Sorrentino
}

Received: 31 October 2007 / Accepted: 14 January 2008 / Published online: 20 February 2008

(C) Springer-Verlag 2008

\begin{abstract}
Aims/hypothesis In patients with type 2 diabetes, reduced levels of circulating endothelial progenitor cells have been reported and these have been correlated with disease severity. In this study, we examined a panel of markers widely used to identify progenitor and/or stem cells, and determined their association with disease severity in diabetic patients. Since expression of chemokine (C-X-C motif) receptor 4 (CXCR4) has been associated with mobilisation and recruitment of progenitor cells, CXCR4 expression was also analysed. Methods Peripheral blood mononuclear cells (PBMCs) from 98 patients with type 2 diabetes and 39 control
\end{abstract}

Electronic supplementary material The online version of this article (doi:10.1007/s00125-008-0939-6) contains supplementary material, which is available to authorised users.

C. G. Egan $\cdot$ R. Lavery $\cdot$ V. Sorrentino $(\bowtie)$

Molecular Medicine Section,

Department of Neuroscience, University of Siena,

via A. Moro,

53100 Siena, Italy

e-mail: v.sorrentino@unisi.it

C. G. Egan · R. Lavery $\cdot$ F. Caporali $\cdot$ V. Sorrentino

Center for Stem Cell Research, University of Siena,

Siena, Italy

F. Caporali $\cdot$ F. Laghi-Pasini

Section of Clinical Immunology,

Department of Clinical Medicine and Immunological Sciences,

University of Siena,

Siena, Italy

C. Fondelli $\cdot$ F. Dotta

Diabetes Unit, Department of Internal Medicine,

Endocrine and Metabolic Sciences and Biochemistry,

University of Siena,

Siena, Italy individuals were analysed by flow cytometry for surface marker expression.

Results Cells expressing different combinations of progenitor and/or stem cell markers were severely reduced in PBMCs of diabetic patients compared with those of control participants. Moreover, a number of these putative progenitor cell populations were negatively associated with disease severity. Reduced expression of CXCR4 and CD34/CXCR4-positive cells was also observed in diabetic patients. PBMCs expressing CXCR4 positively correlated with levels of progenitor cells in control participants but not in diabetic patients. Levels of putative progenitor and CXCR4-positive cells were further decreased in patients with diabetic complications, including cardiovascular and microvascular diseases.

Conclusions/interpretation A generalised decrease in a range of progenitor cell populations was observed in type 2 diabetic patients. This reduction was also negatively associated with disease severity.

Keywords Chemokine (C-X-C motif) receptor 4 - CXCR4 . Endothelial progenitor cells - EPC · Microvascular disease PBMCs · Peripheral blood mononuclear cells · Stem cells . Surface marker - Type 2 diabetes

$\begin{array}{ll}\text { Abbreviations } \\ \text { CVD } & \text { cardiovascular disease } \\ \text { CXCR4 } & \text { chemokine (C-X-C motif) receptor } 4 \\ \text { EPC } & \text { endothelial progenitor cell } \\ \text { KDR } & \text { kinase insert domain receptor } \\ \text { MVD } & \text { microvascular disease } \\ \text { PBMC } & \text { peripheral blood mononuclear cell } \\ \text { SDF-1 } & \text { stromal-derived factor-1 } \\ \text { vWF } & \text { von Willebrand factor }\end{array}$




\section{Introduction}

Type 2 diabetes is a complex metabolic disorder associated with increased risk of cardiovascular disease (CVD) [1-3]. These complications appear to result from a generalised vascular dysfunction resulting in the development of a vasculopathy that, although poorly understood, is likely to arise from damage and dysfunction of the endothelial lining $[1,4-6]$. Increasing evidence indicates that in healthy individuals, circulating endothelial progenitor cells (EPCs) represent a population of bone marrow-derived cells responsible for repairing damaged vascular endothelium and initiating neovasculogenesis [7]. EPCs are primarily identified by the expression of combinations of cell surface antigenic markers, including haematopoietic stem cell markers CD34, CD133 and/or the endothelial cell marker kinase insert domain receptor (KDR, also known as VEGFR2) in different combinations, including CD133/KDR, CD34/KDR and CD34/CD133/KDR [8-18]. However, the precise origin, phenotypic identification and differentiative potential of EPCs is still being debated [10,11]. Moreover, it is possible that different combinations of antigenic markers can identify distinct subsets of EPCs $[9,12,13]$.

Changes in the number of circulating EPCs have been reported in different pathological states and have been associated with endothelial dysfunction and impaired collateral development. For example, the number of EPCs and their migratory activity are both decreased in patients with chronic CVD [13-15], rheumatoid arthritis [16] and pulmonary disease [17]. Reduced levels of circulating EPCs have also been detected in patients affected by type 1 and type 2 diabetes mellitus [18-20]. Conversely, in patients with acute vascular disease, such as acute myocardial infarction, a rapid increase in circulating EPCs has been documented [21]. Accordingly, studies in animal models have shown that circulating EPCs can contribute to therapeutic reendothelialisation and/or neovascularisation, which has raised considerable interest in their use in human therapy $[7,22]$. Evidence indicates that a variety of chemokines, cytokines, growth factors and their specific receptors may regulate the mobilisation and homing of EPCs from bone marrow to the periphery, in addition to processes of proliferation and differentiation $[8,9]$. Chemokine (C-X-C motif) receptor 4 (CXCR4), the receptor for stromal-derived factor-1 (SDF-1), plays an important role in the mobilisation of cells from the bone marrow [23] and may also regulate the mobilisation and recruitment of EPCs [23-27].

Given the absence of a standard for the definition of 'circulating EPCs', we decided to examine a range of different combinations of surface markers known to recognise circulating progenitors and stem cells $[9,11]$ in order to verify whether this may help identify better surrogate markers of disease severity in type 2 diabetes. In addition, this would allow an improved assessment of the levels of putative EPCs with respect to specific complications of diabetes. In our study, the analysis of levels of CXCR4 expression in peripheral blood mononuclear cells (PBMCs) of diabetic patients was included, due to the important role of this receptor in the mobilisation/recruitment of circulating progenitor cells.

Our findings indicate that among the panel of antigenic combinations examined, five of these combinations, including CD34/KDR, CD34/CD31, CD34/CD117, CD34/ CD133 and CD34/CD133/KDR, identify cell populations that best reflect the status of disease severity in patients with type 2 diabetes. A reduced expression of CXCR4 was observed in PBMCs of diabetic patients. The number of CXCR4-positive cells correlated with levels of progenitor cells in healthy control participants but not with those in diabetic patients and was further decreased in patients with cardiovascular and microvascular disease (MVD).

\section{Methods}

Patient characteristics Blood samples were collected from patients referred to the Diabetes Unit at the University Hospital of Siena, Italy. Clinical data for control participants and patients are presented in Table 1. Ninety-eight patients were diagnosed with type 2 diabetes. Diagnosis of type 2 diabetes was the only criterion for inclusion in this study, whereas exclusion criteria included diagnosis of type 1 diabetes and presence of any of the following selfreported medical conditions: auto-immune diseases, current or prior cancers, acute or chronic infection and any other unrelated disease. Diagnosis of diabetes was according to criteria of the American Diabetes Association [28] or the National Cholesterol Education Program [29]. We recruited 39 healthy individuals, who had neither a history of diabetes, cardiovascular, inflammatory or autoimmune disease, nor obesity nor any other chronic disease, as healthy reference controls. This group was matched for age and sex, and presented for the sole purpose of donating blood samples for this study. Ethics committee approval and written informed consent from all participants were obtained.

Vascular complications associated with diabetes included CVD and MVD. CVD was defined as diseases of the circulatory system including cardiac, cerebral or peripheral atherosclerosis. CVD diagnostic criteria were as previously described [30]. MVD was considered to be present in patients presenting with any of the following complications: neuropathy, retinopathy or nephropathy. Diagnostic criteria for neuropathy, diabetic retinopathy and diabetic nephropathy were as described [31-33]. 
Table 1 Healthy control and type 2 diabetic patient characteristics

\begin{tabular}{|c|c|c|}
\hline Characteristic & $\begin{array}{l}\text { Controls } \\
(n=39)\end{array}$ & $\begin{array}{l}\text { Type } 2 \text { diabetes } \\
(n=98)\end{array}$ \\
\hline \multicolumn{3}{|l|}{ General } \\
\hline Age (years) & $56.5 \pm 2.1$ & $60.9 \pm 1.1$ \\
\hline Male patients $n(\%)$ & $22(56.4)$ & $62(63.27)$ \\
\hline BMI $\left(\mathrm{kg} / \mathrm{m}^{2}\right)$ & $24.5 \pm 1.6$ & $29.12 \pm 0.63 * * *$ \\
\hline Disease duration (years) & - & $11.7 \pm 0.9$ \\
\hline \multicolumn{3}{|l|}{ Laboratory } \\
\hline Plasma glucose $(\mathrm{mmol} / \mathrm{l})$ & - & $7.48 \pm 0.19$ \\
\hline $\mathrm{HbA}_{1 \mathrm{C}}(\%)$ & - & $7.1 \pm 0.12$ \\
\hline $\operatorname{AER}(\mathrm{mg} / 24 \mathrm{~h})$ & - & $21.6 \pm 3.3$ \\
\hline Total cholesterol (mmol/l) & - & $4.8 \pm 0.08$ \\
\hline LDL-cholesterol (mmol/1) & - & $2.7 \pm 0.07$ \\
\hline HDL-cholesterol (mmol/l) & - & $1.37 \pm 0.03$ \\
\hline Triacylglycerol (mmol/l) & - & $1.56 \pm 0.09$ \\
\hline \multicolumn{3}{|l|}{ Anamnesis } \\
\hline Hypertension, $n(\%)$ & - & $58(59.2)$ \\
\hline CVD, $n(\%)$ & - & $30(30.6)$ \\
\hline Retinopathy, $n(\%)$ & - & $29(29.6)$ \\
\hline Nephropathy, $n(\%)$ & - & $22(22.45)$ \\
\hline Neuropathy, $n(\%)$ & - & $17(17.4)$ \\
\hline Smoking, $n(\%)$ & $12(30.8)$ & $37(37.8)$ \\
\hline \multicolumn{3}{|l|}{ Medication } \\
\hline Insulin, $n(\%)$ & - & $15(15.3)$ \\
\hline Diet, $n(\%)$ & - & $10(10.2)$ \\
\hline Oral hypoglycaemic agents, $n(\%)$ & - & $67(68.36)$ \\
\hline Combination, $n(\%)$ & - & $6(6.12)$ \\
\hline Statin, $n(\%)$ & - & $30(30.6)$ \\
\hline Aspirin, $n(\%)$ & - & $24(24.5)$ \\
\hline Anti-hypertensive, $n(\%)$ & - & $60(61.2)$ \\
\hline Other, $n(\%)$ & - & $31(31.6)$ \\
\hline
\end{tabular}

Data expressed as $n(\%)$ or mean \pm SEM. Comparisons were made by two-tailed Student's unpaired $t$ test or Mann-Whitney test, for nonparametric data. Laboratory and diagnostic assays were from fasted blood samples; smoking refers to previous or current smokers $* * * p<0.001$

Clinical characteristics and threshold values Patients were further subdivided into two groups (high or low) for being either above or below a threshold value or being positive or negative for a specific complication or characteristic. Threshold values were based upon medical guidelines [28, 29]. Threshold values for continuous variables included age, disease duration, BMI, $\mathrm{HbA}_{1 \mathrm{c}}$, AER, plasma glucose, total cholesterol, triacylglycerol, LDL-cholesterol and HDL-cholesterol, and were calculated from the median value, which also therefore ensured similar patient numbers within the high and low groups. Briefly, this procedure divided all patients according to: age $<$ or $\geq 65$ years; disease duration $<$ or $\geq 10$ years; $\mathrm{BMI}<$ or $\geq 30 \mathrm{~kg} / \mathrm{m}^{2}$; $\mathrm{HbA}_{1 \mathrm{c}},<$ or $\geq 7 \%$; AER $<$ or $\geq 30 \mathrm{mg} / 24 \mathrm{~h}$; plasma glucose $<$ or $\geq 7 \mathrm{mmol} / 1$; total cholesterol $<$ or $\geq 5.2 \mathrm{mmol} / \mathrm{l}$; triacylglycerol $<$ or $\geq 1.7 \mathrm{mmol} / 1$; LDL-cholesterol $<$ or $\geq 2.6 \mathrm{mmol} / \mathrm{l}$; and HDL-cholesterol $>$ or $\leq 1 \mathrm{mmol} / \mathrm{l}$ for men and $>$ or $\leq 1.3 \mathrm{mmol} / 1$ for women. Dichotomous variables (i.e. positive or negative) included CVD, hypertension and MVD. With regard to patients with MVD, a positive score was given to patients presenting with at least one of the three complications considered (retinopathy, neuropathy or nephropathy). Dyslipidaemia was considered present if patients exceeded the threshold value for at least one out of four parameters (e.g. total cholesterol, triacylglycerol, LDL-cholesterol or HDL-cholesterol). Other characteristics included: sex (male sex considered a clinical characteristic) and smoking. Sex, age and smoking were considered generic factors, while all other factors were specific to the patient cohort.

Isolation of peripheral blood mononuclear cells Blood samples were obtained from a forearm vein and processed immediately. Blood samples were diluted 1:1 in Dulbecco's PBS (Sigma-Aldrich, Milan, Italy) and cells were then separated on a Ficoll-Paque PLUS (Amersham Biosciences, Uppsala, Sweden) gradient at $400 \mathrm{~g}$. PBMCs were collected, washed in PBS and subjected to low-speed centrifugation to remove platelets. The PBMC pellet was resuspended in $2 \mathrm{ml}$ Dulbecco's PBS.

Flow cytometry Flow cytometry was used to determine the percentage of cells expressing respective cell surface antigens. Briefly, aliquots of $5 \times 10^{5}$ PBMCs were stained with anti-mouse monoclonal antibodies. Staining was performed according to manufacturer's instructions (BD Biosciences, Franklin Lakes, NJ, USA) for $30 \mathrm{~min}$ at $4^{\circ} \mathrm{C}$. PBMCs were analysed for the expression of cell surface antigens with direct two- or three-colour analysis using monoclonal antibodies recognising the following different cell surface antigens: allophycocyanin-conjugated CD34; phycoerythrin-conjugated CD117 and CXCR4 (all from BD Biosciences, Franklin Lakes, NJ, USA); CD133 (Miltenyi Biotech, Bergisch Gladbach, Germany); CD31 and VEcadherin (both from Santa Cruz Biotechnology, Santa Cruz, CA, USA); fluorescein isothiocyanate-conjugated CD14 (BD Biosciences) and CD45 (Santa Cruz Biotechnology); and KDR and von Willebrand factor (vWF; both from Sigma-Aldrich) followed by fluorescein isothiocyanateconjugated secondary antibody (BD Biosciences). Eight of these markers were also analysed in double combination and one in triple combination, in the cohort of patients and healthy control participants. Identical IgG isotypes served as negative controls (BD Biosciences). For triple labelling experiments, standard electronic compensation was performed manually using CellQuest Software (BD Biosciences). After incubation, cells were washed with PBS and analysed on a FACS Calibur flow cytometer (BD Biosciences) with CellQuest Software. Each analysis consisted of between 100,000 and 200,000 events. Threshold was 
adjusted in forward and side scatter dot plot to exclude cellular debris. Monocytic and lymphocytic populations were identified by size and light-scattering properties [34]. CD14 and CD45 staining was used to further verify the presence of monocytic and lymphocytic cell populations, respectively. To exclude the possibility that enumeration may have been influenced by changes in monocyte and/or lymphocyte number (and/or ratio), we performed differential blood counts for control participants and diabetic patients. Further confirmation of PBMC number by FACS analysis using CD14 and CD45 revealed no difference in monocyte/lymphocyte cell number between the two groups. Three different investigators, blinded to the sample origin, conducted FACS analysis (C. G. Egan, R. Lavery and F. Caporali).

Statistical analysis Data are presented as mean \pm SEM. Statistical analysis was performed using Instat Software (GraphPad, San Diego, CA, USA) or MedCalc Software (Broekstraat, Mariakerke, Belgium). Data were initially evaluated for normal distribution with the KolmogorovSmirnov test. Not normally distributed continuous variables were compared by the Mann-Whitney test. Comparisons between two groups with normally distributed variables were analysed by Student's unpaired $t$ test. Comparisons between three or more groups were analysed by one-way ANOVA followed by Dunnett's post hoc. The Hochberg procedure was applied to account for $\alpha$-inflation by type 1 error derived from multiple testing. Parametric linear correlations (between CXCR4-positive cells and progenitor cells) were assessed by Pearson's correlation coefficient $(r)$, whereas non-parametric correlations (between disease severity score and progenitor cells) were examined by Spearman's rank correlation coefficient (rho). Multivariate analysis by multiple regression was applied to reveal associations between clinical characteristics (independent variables) and progenitor cells (dependent variable). Receiver operating characteristic curve analysis was applied to determine whether progenitor populations could be used to discriminate between healthy control participants and diabetic patients. Where comparisons were made, quoted $p$ values are two-tailed; $n$ values refer to the number of patients examined. A value of $p<0.05$ was considered statistically significant.

\section{Results}

Patients and control participants Among the 98 patients studied, 13 presented without any complication, 39 had one complication and 46 had two or more complications associated with type 2 diabetes. Neither age nor sex were significantly different in the diabetic group when compared with controls. BMI, however, was higher in the patient group compared with healthy control participants.

Flow cytometric analysis of circulating progenitor cells in PBMCs FACS analysis performed on total PBMCs revealed a significant reduction in the number of cells expressing most of the markers analysed in diabetic patients compared with control individuals. This reduction was observed in the number of cells co-expressing CD34/KDR, CD117/KDR，CD133/KDR，CXCR4/KDR，CD34/CD31 and CD34/CD117, as well as in cells positive for CD34/ CD133/KDR (Table 2). In diabetic patients we also noted a reduced number of cells expressing markers known to be present on circulating endothelial cells, including KDR and VE-cadherin, in addition to markers expressed on progenitor cells, such as CD117, CD34 and CD133. A reduction in the number of CXCR4 and CD34/CXCR4-positive cells was also observed in PBMCs of diabetic patients. Receiver operating characteristic curve analysis revealed that CD34/ CD117-positive cells could discriminate between control and diabetic patients $(p=0.0047$, data not shown). A priori internal control was to include markers recognising cells that are not progenitor phenotypes, such as CD31, vWF and KDR, which would not be expected to change between the two groups. Although KDR was reduced in diabetic patients, levels of both CD31 and vWF-positive cells (total PBMCs) were similar in both groups. The percentage of cells labelled in the total PBMC population was not equally distributed within the lymphocytic and monocytic populations. Among the 14 subsets that were reduced in the total population, five were reduced in the lymphocytic population while nine were reduced in the monocytic population (Table 2). Since the total PBMC population represents both lymphocytic and monocytic populations, this cell population should best reflect a more comprehensive analysis. We therefore used data derived from total cell population in all further analysis.

Clinical characteristics are negatively correlated with cells expressing progenitor markers We next wanted to evaluate whether the levels of cells expressing some of the marker combinations used within the diabetic cohort were associated with clinical characteristics (Table 2). To generate a severity score, diabetic patients were scored positive or negative (or exceeding a threshold value) for a clinical characteristic as detailed above and also as previously described [18]. Thus, patients scoring positive for all clinical characteristics would theoretically have a disease severity score of 12 . Univariate correlation regression analysis was performed between levels of cells identified by FACS analysis and number of clinical characteristics (Fig. 1). This analysis revealed that among the 17 different surface markers examined, five marker combinations 


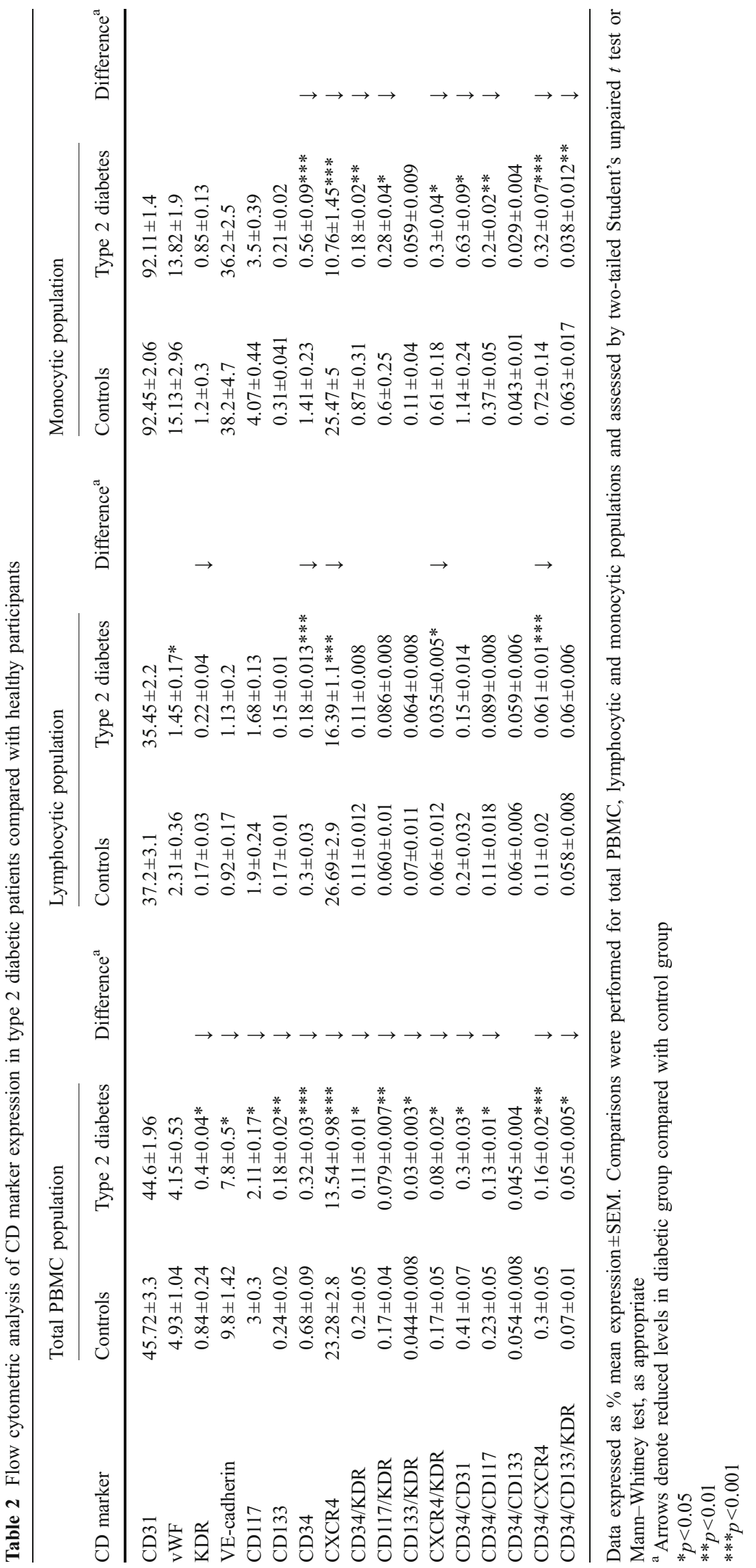



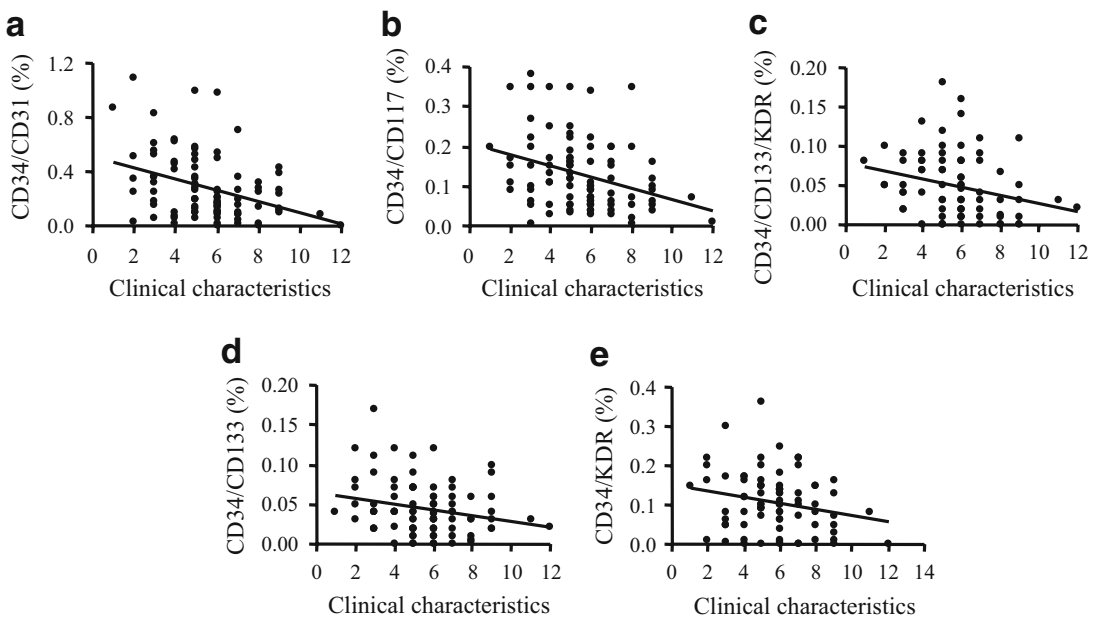

Fig. 1 Clinical characteristics are negatively correlated with cells expressing progenitor markers. Levels of progenitor cells from the total PBMC population of patients with type 2 diabetes were shown to be negatively correlated with disease severity score. Severity score is based on 12 different clinical characteristics (see Methods). Univariate

showed a negative correlation with the overall disease severity score. These combinations identified cells coexpressing CD34/CD31, CD34/CD117, CD34/CD133/ KDR, CD34/CD133 and CD34/KDR (Fig. 1).

Impact of individual clinical characteristics upon levels of progenitor cells Having first identified a panel of cell surface markers that showed a negative association with overall disease severity (Fig. 1), we next wanted to further examine the relative impact of individual clinical characteristics on these cells (Fig. 2, Electronic supplementary material $[\mathrm{ESM}]$ Tables 1 and 2). This was examined in two ways: (1) by subgroup analysis of patients based on threshold values, followed by unpaired $t$ test; and (2) by multivariate regression analysis. Patients were subdivided into high and low groups based on threshold values, as indicated above (Methods). The Hochberg procedure was then applied to account for type 1 error inflation and differences between high and low groups were arranged in order of level of statistical significance (high significance to low significance) as depicted in Fig. 2. This analysis revealed that of the five progenitor populations examined, CD34/CD133/KDR appeared to be the most sensitive marker, since it was significantly decreased in patients presenting with MVD compared with patients without MVD ( $p=0.0016)$ (Fig. 2c). In comparison to the other clinical characteristics examined, MVD, high $\mathrm{HbA}_{1 \mathrm{c}}$ and CVD were the clinical characteristics that were more frequently associated with a reduction in levels of progenitor cells as evidenced by their relative position in order.

Multivariate analysis was then applied to examine the association between clinical characteristics and progenitor correlation was performed using Spearman's rank correlation coefficient (rho). $p<0.05$ was considered statistically significant. a CD34/ $\mathrm{CD} 31$, rho $=-0.36, p=0.0004$; (b) $\mathrm{CD} 34 / \mathrm{CD} 117$, rho $=-0.32, p=0.002$; (c) $\mathrm{CD} 34 / \mathrm{CD} 133 / \mathrm{KDR}$, rho $=-0.25, p=0.028$; (d) $\mathrm{CD} 34 / \mathrm{CD} 133$, rho $=$ $-0.23, p=0.033$; (e) CD34/KDR, rho $=-0.22, p=0.048$

cells. Collectively, clinical characteristics did not reveal any association between the progenitor cells (ESM Table 1). When clinical characteristics were separated into risk factors and clinical complications, a significant association was noted between patients with clinical complications and CD34/CD31 and CD34/CD133/KDR-positive cells (ANOVA, $p=0.029$ and $p=0.039$, respectively). In addition, patients with MVD were significantly associated with CD34/CD117 and CD34/CD133/KDR-positive cells $(p=0.035$ and $p=$ 0.048, respectively; ESM Table 2).

We next wanted to verify whether type 2 diabetic patients with at least two of the three complications (CVD, elevated $\mathrm{HbA}_{1 \mathrm{c}}$ or MVD) had a significant reduction in the circulating progenitor cells identified in Fig. 2, compared with patients negative for these complications. As shown in Fig. 3a, a reduction in the levels of cells expressing four of the candidate markers examined was even more pronounced in patients with two of the three complications $(n=38)$ compared with patients without these complications $(n=21)$. We also noted that patients with a combination of two of either CVD, high $\mathrm{HbA}_{1 \mathrm{c}}$ or MVD had a significant reduction in CXCR4-positive cells $(9.7 \pm 1.04$ compared with $16.11 \pm 2.12 \%, p=0.0037$; Fig. 3b). Since patients positive for MVD also showed reduced levels of progenitor cells (Fig. 2), we wanted to further examine the number of these progenitor cells in specific complications associated with MVD. Patients with nephropathy did not have any further reduction in progenitor cell populations. However, with respect to patients without MVD, a significant reduction in four combinations of surface markers was observed in those presenting with retinopathy (Fig. 3c). In addition, patients with neuropathy revealed a significant reduction in levels of two of the cell populations examined. 
Fig. 2 a-e Impact of individual clinical characteristics upon levels of progenitor cells as indicated. Clinical characteristics were divided into subgroups based upon criteria described in Methods. Student's unpaired $t$ test or Mann-Whitney test was used to compare high/positive (black bars) or low/negative (white bars) subgroups. The Hochberg procedure was then applied to account for type 1 error inflation. $\dagger p=0.0016$, following Hochberg correction (c). Data are presented as \% mean \pm SEM. Dis Dur, disease duration; Hyp, hypertension

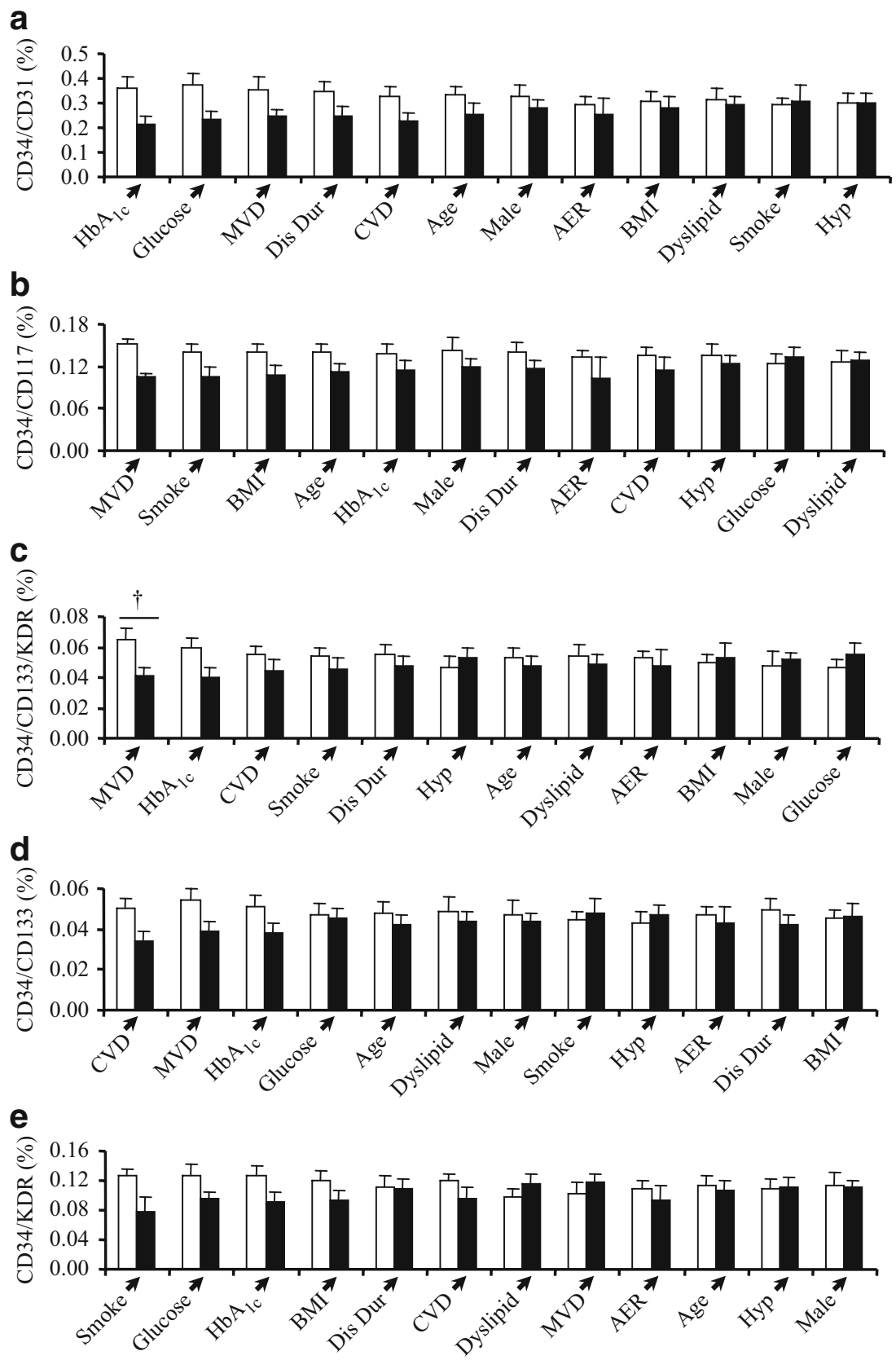

Interestingly, the number of CXCR4-positive cells was significantly lower in patients with retinopathy than in patients without MVD complications (9.6 \pm 1.4 compared with $15.4 \pm 1.4 \%, p=0.0072$, one-way ANOVA $p=0.039$; Fig. 3d).

Correlation of CXCR4 expression with levels of progenitor cells in healthy control and type 2 diabetic groups The decrease in CXCR4-expressing cells observed in diabetic patients (Table 2) prompted us to examine the relationship between the number of PBMCs expressing CXCR4 and the number of cells expressing progenitor and/or stem cell surface markers that negatively correlated with disease severity score in Fig. 1 and that were reduced in patients with vascular complications (Figs. 2 and 3). In control individuals, a positive correlation was observed between the number of CXCR4-positive cells and that of progenitor cells examined (Table 3). In contrast, no correlation was detected in type 2 diabetic patients.

\section{Discussion}

The growing interest in EPCs stems from their potential role in the maintenance of endothelial integrity, function 

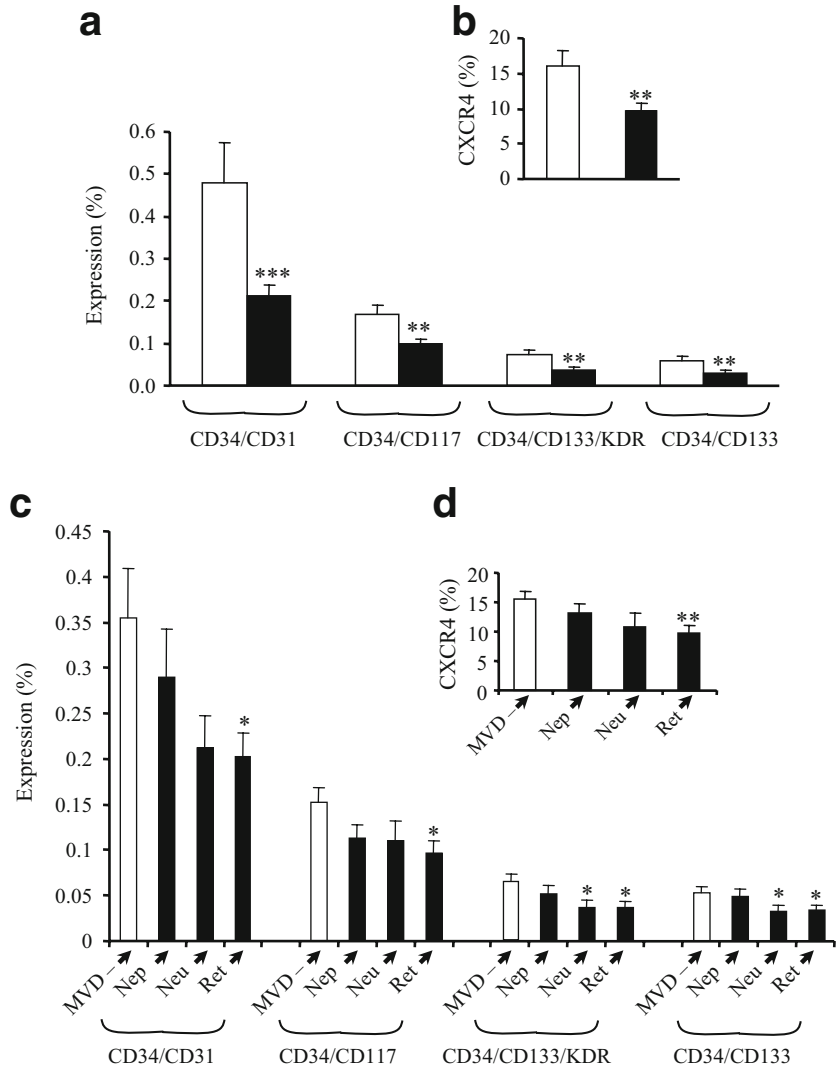

Fig. 3 Effect of $\mathrm{CVD}, \mathrm{HbA}_{1 \mathrm{c}}$ and $\mathrm{MVD}$ on levels of PBMCs expressing putative EPC markers. a Patients presenting with two of either MVD, CVD and high levels of $\mathrm{HbA}_{1 \mathrm{c}}$ (black bars; $n=38$ ) showed a marked reduction in circulating putative EPCs and total PBMCs expressing CXCR4 (b), compared with patients without any of these complications (white bars; $n=21$ ). c Subgroup analysis of the MVD group identified both neuropathy (Neu; $n=17$ ) and retinopathy (Ret; $n=29$ ) as complications whereby progenitor cell levels were further decreased in type 2 diabetic patients. d Expression of CXCR4 in patients with MVD complications as indicated, compared with patients negative for MVD $(n=46)$. Data are presented as $\%$ mean \pm SEM. In all cases, comparisons were made between positive (black bars) and negative subgroups (white bars), using Student's unpaired $t$ test or Mann-Whitney test or ANOVA followed by Dunnett's post hoc, as appropriate. ANOVA revealed significant differences between MVD subgroups for all four markers examined (CD34/CD31, $p=0.047$; CD34/CD117, $p=0.036$; CD34/CD133/KDR, $p=0.026$; CD34/CD133, $p=0.043) .{ }^{*} p<0.05,{ }^{*} p<<0.01,{ }^{*} * *<<0.001$. Nep, nephropathy; Neu, neuropathy; Ret, retinopathy

and postnatal neovascularisation. In addition, accumulating evidence indicates a reduced availability and impaired EPC function in several pathologies characterised by cardiovascular damage [13-15]. Other studies are also providing encouraging, yet preliminary insights into the potential use of EPCs in the clinical setting [9, 15, 35]. Recent reports have shown that EPCs are functionally impaired in diabetic patients [19, 20, 36]. In addition, depletion of EPCs, defined by co-expression of CD34/KDR in cells from peripheral blood, was observed in type 2 diabetic patients with respect to non-diabetic controls and this reduction in
EPC levels was also found to negatively correlate with disease severity score in diabetic patients [18]. The purpose of this study was to examine a wide panel of antigenic markers known to be present on both haematopoietic cells and EPCs in order to determine the extent of progenitor cell depletion in type 2 diabetes and its association with disease severity. Our findings reveal that type 2 diabetic patients present a generalised deficit in availability of circulating progenitor cells, regardless of whether they are haematopoietic progenitors or subsets of EPCs. These progenitor populations include cells positive for CD34/KDR, which may represent early EPCs [18], and cell populations expressing both haematopoietic (CD133 and CD117) and endothelial lineage (KDR) markers (CD133/KDR or CD117/KDR-expressing cells), which may also represent early EPCs. On the other hand, cells expressing CD34/ CD31 may represent a population of late EPCs. Additional cells expressing markers that recognise haematopoietic progenitors, such as CD34/CD117-positive cells, were also reduced in diabetic patients. In this respect, it is worth noting that the phenotypic characterisation of circulating progenitors and/or EPCs is still being debated [10]. For example, EPCs recognised by the expression of CD34/ CD133/KDR, which were also reduced in this study, have recently been proposed to represent haematopoietic rather than endothelial progenitors [11]. In fact, partly due to their limited number, functional information on EPCs is largely missing. Clearly, a better characterisation of the different progenitor cells, based on in vivo test, is required. However, this was beyond the scope of the present study, and in the absence of such a characterisation, we therefore prefer to refer to the progenitor populations identified as 'putative' EPCs.

Despite the lack of information on the biological properties of the progenitor cells identified in both ours and other studies, it is clearly evident that diabetic patients have reduced levels of circulating progenitors and that this is more pronounced in patients with an increased number of clinical characteristics. It is worth noting, however, that even in diabetic patients without complications, levels of

Table 3 Correlation of CXCR4 expression with levels of progenitor cells in healthy control participants and in type 2 diabetic patients

\begin{tabular}{llc}
\hline CD marker & Controls & Type 2 diabetes \\
\hline CD34/CD31 & $0.38^{*}$ & -0.03 \\
CD34/CD117 & $0.38^{*}$ & 0.05 \\
CD34/CD133/KDR & $0.35^{*}$ & 0.11 \\
CD34/CD133 & $0.38^{*}$ & 0.22 \\
CD34/KDR & $0.43^{* *}$ & -0.18 \\
\hline
\end{tabular}

Data expressed as correlation coefficient values $(r)$. Correlations were assessed by Pearson's correlation

$* p<0.05$

$* * p<0.01$ 
the majority of progenitor cells were still significantly depleted, when compared with those of control individuals. Indeed, we noted that levels of selected putative EPCs were reduced in patients with high levels of $\mathrm{HbA}_{1 \mathrm{c}}$, glucose and CVD, confirming previous findings [18-20, 37]. We also noted a generalised reduction in putative EPCs in patients with MVD. In addition, analysis of putative EPC levels in patients with MVD revealed further differences between patients with nephropathy, who did not exhibit altered levels of putative EPCs, and patients with retinopathy or neuropathy, who revealed a pronounced reduction in putative EPCs, compared with patients without these complications. In agreement with a potential pathogenic role of the observed reduction of EPCs in patients with diabetic neuropathy, it has been shown in a rat model of this pathology that administration of EPCs improved nerve conduction and blood flow [38]. In contrast to diabetic neuropathy, which is characterised by microvascular dysfunction, patients with diabetic retinopathy present significant capillary proliferation in the retina [36]. We report here a significant reduction in putative EPCs in patients with diabetic retinopathy, a finding which agrees with some, but not all, published studies [37, 39, 40]. How angiogenesis can be increased in the presence of reduced EPC levels in these patients is not clear and actually represents a paradox [39]. However, it is interesting to note that in the vitreous humour of patients with severe diabetic retinopathy, levels of SDF-1, the ligand of CXCR4 receptor, are increased and that neutralising antibody to SDF-1 prevented retinal neovascularisation in a murine model of diabetic retinopathy [41]. It could therefore be hypothesised that increased concentrations of SDF-1 may still be sufficient to recruit EPCs to the retina to support microvascular proliferation despite depletion of EPCs in the peripheral circulation.

Our study also aimed to determine the levels of CXCR4positive PBMCs in diabetic patients. In fact, reduced mobilisation of EPCs from bone marrow has been proposed as a possible causative mechanism in some conditions where circulating EPCs are reduced [7, 27]. Among other possible mechanisms for EPC reduction, both levels of SDF-1 and expression of its receptor CXCR4 have been proposed to participate in regulating mobilisation and/or recruitment of progenitor cells $[23,25,27]$. Our data indicate a significant reduction of CXCR4 expression in PBMCs of patients with type 2 diabetes.

A reduction in PBMCs expressing CXCR4 has also been observed in patients with CVD $[42,43]$, suggesting that the decrease in CXCR4 expression, in the presence of reduced levels of EPCs, may not be restricted to type 2 diabetic patients. Moreover, we have identified that the number of CXCR4-expressing cells positively correlated with levels of progenitor cells in control individuals, while this correlation was absent in diabetic patients. The number of CXCR4expressing cells was further decreased in patients with cardiovascular and microvascular complications. In line with a potential role of CXCR4 signalling in EPCs, a reduced responsiveness to SDF-1 has been shown in EPCs from patients with coronary artery disease [27]. More recently, it has been reported that diabetic patients have defective CD34+ migration in response to SDF-1 and/or vascular endothelial growth factor [44]. Consistent with these reports, SDF-1 genotype has been shown to influence insulin-dependent mobilisation of adult progenitor cells in type 2 diabetes [45]. In diabetic rats a reduced mobilisation of EPCs from bone marrow after hindlimb ischaemia has been reported [46] and this impaired mobilisation has been shown to be reversed by SDF-1 [47]. Collectively, data reported in the literature and the results presented here suggest that a reduction in CXCR4 expression and/or function may in part, contribute to reduced levels of circulating EPCs in diabetes.

In summary, we have found that different populations of cells expressing progenitor markers are reduced in type 2 diabetes. A number of these cell populations may represent putative EPCs and therefore may be considered as valuable biomarkers for assessing disease severity, specifically in the context of cardiovascular and microangiopathic-associated complications. Type 2 diabetic patients also had a reduction in cells expressing CXCR4 and CD34/CXCR4. Future studies investigating the biological properties of these candidate EPCs are needed to improve our understanding of EPC biology and their role in human diseases, and hopefully to contribute to the development of novel therapeutic strategies.

Acknowledgements We thank S. Rossi, University of Siena, for statistical advice. This work was supported by a grant from Regione Toscana to the Centre for Stem Cell Research, University of Siena.

Duality of interest The authors declare that there is no duality of interest associated with this manuscript.

\section{References}

1. Waltenberger J (2001) Impaired collateral vessel development in diabetes: potential cellular mechanisms and therapeutic implications. Cardiovasc Res 16:554-560

2. St Clair L, Ballantyne CM (2007) Biological surrogates for enhancing cardiovascular risk prediction in type 2 diabetes mellitus. Am J Cardiol 99:80-88

3. Stumvoll M, Goldstein BJ, van Haeften TW (2005) Type 2 diabetes: principles of pathogenesis and therapy. Lancet 365:1333-1346

4. Landmesser U, Drexler H (2005) The clinical significance of endothelial dysfunction. Curr Opin Cardiol 20:547-551

5. Deanfield JE, Halcox JP, Rabelink TJ (2007) Endothelial function and dysfunction: testing and clinical relevance. Circulation 115:1285-1295 
6. Vita JA (2005) Endothelial function and clinical outcome. Heart 91:1278-1279

7. Takahashi T, Kalka C, Masuda H et al (1999) Ischemia- and cytokine-induced mobilization of bone marrow-derived endothelial progenitor cells for neovascularization. Nat Med 5:434-438

8. Asahara T, Murohara T, Sullivan A et al (1997) Isolation of putative progenitor endothelial cells for angiogenesis. Science 275:964-967

9. Hristov M, Wolfgang W, Weber PC (2003) Endothelial progenitor cells-mobilization differentiation and homing. Arterioscler Thromb Vasc Biol 23:1185-1189

10. Ingram DA, Caplice NM, Yoder MC (2005) Unresolved questions, changing definitions, and novel paradigms for defining endothelial progenitor cells. Blood 106:1525-1531

11. Case J, Mead LE, Bessler WK et al (2007) Human CD34+ AC133+VEGFR-2+ cells are not endothelial progenitor cells but distinct, primitive hematopoietic progenitors. Exp Hematol 35:1109-1118

12. Hur J, Yoon CH, Kim HS et al (2004) Characterization of two types of endothelial progenitor cells and their different contributions to neovasculogenesis. Arterioscler Thromb Vasc Biol 24:288-293

13. Urbich C, Dimmeler S (2004) Endothelial progenitor cells: characterization and role in vascular biology. Circ Res 95:343353

14. Vasa M, Fichtlscherer S, Aicher A et al (2001) Number and migratory activity of circulating endothelial progenitor cells inversely correlate with risk factors for coronary artery disease. Circ Res 89:E1-E7

15. Shantsila E, Watson T, Lip GY (2007) Endothelial progenitor cells in cardiovascular disorders. J Am Coll Cardiol 49:741-752

16. Grisar J, Aletaha D, Steiner CW et al (2005) Depletion of endothelial progenitor cells in the peripheral blood of patients with rheumatoid arthritis. Circulation 111:204-211

17. Fadini GP, Schiavon M, Cantini M et al (2006) Circulating progenitor cells are reduced in patients with severe lung disease. Stem Cells 24:1806-1813

18. Fadini GP, Miorin M, Facco M et al (2005) Circulating endothelial progenitor cells are reduced in peripheral vascular complications of type 2 diabetes mellitus. J Am Coll Cardiol 45:1449-1457

19. Tepper OMGR, Capla JM, Kalka C et al (2002) Human endothelial progenitor cells from type II diabetics exhibit impaired proliferation adhesion and incorporation into vascular structures. Circulation 106:2781-2786

20. Loomans CJ, de Koning EJ, Staal FJ et al (2004) Endothelial progenitor cell dysfunction: a novel concept in the pathogenesis of vascular complications of type 1 diabetes. Diabetes 53:195-199

21. Massa M, Rosti V, Ferrario M et al (2005) Increased circulating hematopoietic and endothelial progenitor cells in the early phase of acute myocardial infaction. Blood 105:199-205

22. Isner JM, Asahara T (1999) Angiogenesis and vasculogenesis as therapeutic strategies for postnatal neovascularization. J Clin Invest 103:1231-1236

23. Kucia M, Ratajczak J, Ratajczak MZ (2005) Bone marrow as a source of circulating CXCR4 + tissue-committed stem cells. Biol Cell 97:133-146

24. Ceradini DJ, Gurtner GC (2005) Homing to hypoxia: HIF-1 as a mediator of progenitor cell recruitment to injured tissue. Trends Cardiovasc Med 15:57-63

25. Ruiz de Almodovar C, Luttun A, Carmeliet P (2006) An SDF-1 trap for myeloid cells stimulates angiogenesis. Cell 124:18-21

26. Yamaguchi J, Kusano KF, Masuo O et al (2003) Stromal cell-derived factor-1 effects on ex vivo expanded endothelial progenitor cell recruitment for ischemic neovascularization. Circulation 107:1322-1328
27. Walter DH, Haendeler J, Reinhold J et al (2005) Impaired CXCR4 signaling contributes to the reduced neovascularisation capacity of endothelial progenitor cells from patients with coronary artery disease. Circ Res 97:1142-1151

28. American Diabetes Association (2007) Standards of medical care in diabetes-2007. Diabetes Care 30(Suppl 1):S4-S41

29. Grundy SM, Cleeman JI, Merz CN et al (2004) Implications of recent clinical trials for the National Cholesterol Education Program Adult Treatment Panel III guidelines. Circulation 110:227-239

30. Miller DD, Shaw LJ (2006) Foreword: cardiovascular disease: diagnostic, prognostic, and measurement paradigms for reducing patient risk. J Cardiovasc Nurs 21(6 Suppl 1):S1

31. Devendra D, Farmer K, Bruce G, Hughes P, Vivian G, Millward BA (2001) Diagnosing osteomyelitis in patients with diabetic neuropathic osteoarthropathy. Diabetes Care 24:2154-2155

32. Cypress M, Tomky D (2006) Microvascular complications of diabetes. Nurs Clin North Am 41:719-736 ix

33. Molitch ME, DeFronzo RA, Franz MJ et al (2004) American Diabetes Association. Nephropathy in diabetes. Diabetes Care Suppl 1:S79-S83

34. Rogler G, Hausmann M, Vogl D et al (1998) Isolation and phenotypic characterization of colonic macrophages. Clin Exp Immunol 112:205-215

35. Wang XX, Zhang FR, Shang YP et al (2007) Transplantation of autologous endothelial progenitor cells may be beneficial in patients with idiopathic pulmonary arterial hypertension: a pilot randomized controlled trial. J Am Coll Cardiol 49:1566-1571

36. Fadini GP, Sartore S, Agostini C, Avogaro A (2007) Significance of endothelial progenitor cells in subjects with diabetes. Diabetes Care 30:1305-1313

37. Kusuyama T, Omura T, Nishiya D et al (2006) Effects of treatment for diabetes mellitus on circulating vascular progenitor cells. J Pharmacol Sci 102:96-102

38. Naruse K, Hamada Y, Nakashima E et al (2005) Therapeutic neovascularization using cord blood-derived endothelial progenitor cells for diabetic neuropathy. Diabetes 54:1823-1828

39. Fadini GP, Sartore S, Baesso I et al (2006) Endothelial progenitor cells and the diabetic paradox. Diabetes Care 29:714-716

40. Lee IG, Chae SL, Kim JC (2006) Involvement of circulating endothelial progenitor cells and vasculogenic factors in the pathogenesis of diabetic retinopathy. Eye 20:546-552

41. Butler JM, Guthrie SM, Koc M et al (2005) SDF-1 is both necessary and sufficient to promote proliferative retinopathy. J Clin Invest 115:86-93

42. Damas JK, Waehre T, Yndestad A et al (2002) Stromal cellderived factor-1alpha in unstable angina: potential antiinflammatory and matrix-stabilizing effects. Circulation 106:36-42

43. Honold J, Lehmann R, Heeschen C et al (2006) Effects of granulocyte colony simulating factor on functional activities of endothelial progenitor cells in patients with chronic ischemic heart disease. Arterioscler Thromb Vasc Biol 26:2238-2243

44. Segal MS, Shah R, Afzal A et al (2006) Nitric oxide cytoskeletalinduced alterations reverse the endothelial progenitor cell migratory defect associated with diabetes. Diabetes 55:102-109

45. Humpert PM, Neuwirth R, Battista MJ et al (2005) SDF-1 genotype influences insulin-dependent mobilization of adult progenitor cells in type 2 diabetes. Diabetes Care 28:934-936

46. Fadini GP, Sartore S, Schiavon M et al (2006) Diabetes impairs progenitor cell mobilisation after hindlimb ischaemia-reperfusion injury in rats. Diabetologia 49:3075-3084

47. Gallagher KA, Liu ZJ, Xiao M et al (2007) Diabetic impairments in NO-mediated endothelial progenitor cell mobilization and homing are reversed by hyperoxia and SDF-1. J Clin Invest $117: 1249-1259$ 